\title{
Chronic hypertrophic cranial pachymeningitis associated with HTLV-I infection
}

\author{
Y Kawano, J Kira
}

\begin{abstract}
Two patients presenting with recurrent multiple cranial neuropathy showed diffuse thickening and gadolinium enhancement of the dura mater on brain MRI. Both had anti-HTLV-I antibodies in serum. A quantitative polymerase chain reaction study of the peripheral blood disclosed that the HTLV-I proviral DNA loads increased considerably in one case and moderately in the other. Both showed a spontaneous proliferation of peripheral blood lymphocytes as well as an increase in helper/inducer $T$ cells. Neither had any other underlying infections or autoimmune diseases. Thus it is possible that hypertrophic pachymeningitis developed as a result of multiorgan involvement of HTLV-I infection in these patients.
\end{abstract}

(F Neurol Neurosurg Psychiatry 1995;59:435-437)

Keywords: hypertrophic cranial pachymeningitis; HTLV-I infection

Chronic hypertrophic cranial pachymeningitis is an extremely rare disease. Several aetiologies, either infection or autoimmunity, have been reported. Most aspects of this disease, however, remain to be elucidated. We describe two cases of hypertrophic pachymeningitis that occurred in an HTLV-I carrier state, in which autoimmune and inflammatory conditions such as HTLV-I associated myelopathy (HAM)/tropical spastic paraparesis (TSP), ${ }^{12}$ arthritis, ${ }^{3}$ myositis, ${ }^{4}$ alveolitis, ${ }^{5}$ uveitis, ${ }^{6}$ and dacryosialadenitis, ${ }^{7}$ have been reported.

\section{Case reports}

PATIENT 1

A 36 year old man noted tingling in his left cheek and left facial weakness followed by double vision and headache in July 1991. The neurological examination at admission disclosed left trigeminal, abducens and facial nerve paresis, and bilateral swollen discs. High dose oral corticosteroids alleviated the multiple cranial neuropathy. In July 1993 the tingling sensation of the left cheek and double vision recurred. A month later gustatory sensation became impaired on the left side of his tongue. Neurologically he had bilateral optic atrophy, left oculomotor nerve paresis, left trigeminal nerve paraesthesia, and left facial nerve paresis.

The following laboratory studies were normal: complete blood count, erythrocyte sedimentation rate, C-reactive protein, serum and urine chemistry, immunoglobulins, C3, C4, $\mathrm{CH} 50$, angiotensin converting enzyme, HbAlc, thyroid hormones, carcinoembryonic antigen, squamous cell carcinoma antigen, and CA19-9. The serum was negative for rheumatoid factor, antinuclear antibody, lupus erythematosus test, anti-ribonucleoprotein, anti-Smith, anti-double strand DNA, SS-A, SS-B, test for syphilis, toxoplasma test, and immune complexes. Anti-HTLV-I antibody was positive at 1:4096 dilution by the particle agglutination (PA) method in serum. A CSF examination showed an increased opening pressure $\left(210 \mathrm{~mm} \mathrm{H}_{2} \mathrm{O}\right)$, but the cell counts, protein, glucose, and IgG index were all normal. Culture of CSF for bacteria and fungi was negative. Anti-HTLV-I antibody was positive at a 1:8 dilution by the PA method. The HTLV-I pX DNA amounts in peripheral blood mononuclear cells were measured by a quantitative polymerase chain reaction analysis and normalised by the amounts of $\beta$-globin DNA as described previously. ${ }^{8}$ The calculated copy numbers of HTLV-I genome increased to 58 copies per 100 peripheral blood mononuclear cells. Spontaneous proliferation of peripheral blood mononuclear cells greatly increased to $7799 \mathrm{cpm}$ (normal 70-700) and the stimulation index to phytohaemagglutinin (PHA) and concavalin A (ConA) significantly decreased to $14 \cdot 8$ (normal 114-358) and $15 \cdot 1$ (normal 80-274), respectively. Flow cytometry of peripheral blood mononuclear cells disclosed an increase of CD4 + cells $(55.6 \%$, normal $33 \cdot 2-51 \cdot 1)$ and a decrease of CD8 + cells $(14 \cdot 1 \%$, normal $19 \cdot 8-33 \cdot 6)$.

On brain MRI, diffuse thickening and gadolinium enhancement of the dura mater in the falx, cranial convexity, skull base, and cerebellar tentorium were evident (figure). An abnormal accumulation of gallium was seen in the parietal convexity by scintigraphy. High dose oral corticosteroids ( $60 \mathrm{mg} /$ day) again alleviated both his symptoms and the gadolinium enhancement of the dura mater.

\section{PATIENT 2}

The patient was a 63 year old man. He presented with a three month history of bilateral 
Coronal gadolinium-DTPA enhanced $T 1$ weighted $M R I$ of patient 1 before $(A)$ and after $(B)$ treatment with corticosteroids. Note the decrease in the gadolinium enhancement of both the falx and the cerebellar tentorium after treatment

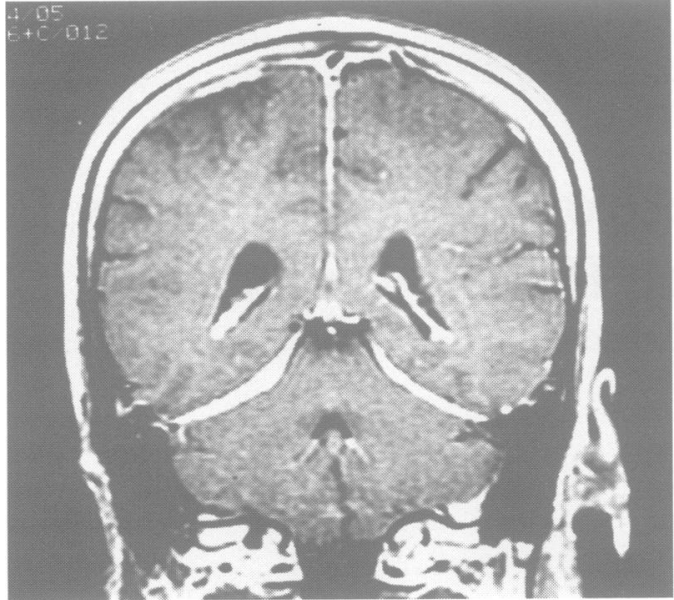

(A)

blurred vision at 43 years of age. Visual acuity again decreased gradually at 54 years of age. Since then he has received chronic treatment with low dose corticosteroids. He has had chronic bronchopneumonia of unknown aetiology since he was 58 years old. He experienced left facial weakness followed by spontaneous recovery three months later at the age of 60 . One year later, a tingling sensation on the left side of his face and hoarseness developed gradually. When he was referred to our clinic, a general physical examination showed moist rales in both lung fields. Neurologically, he had bilateral anosmia, bilateral optic atrophy, left trigeminal nerve hypaesthesia, and hypalgesia with absent corneal reflex, atrophy of left masseter muscle, left abducens nerve paresis, and hoarseness.

The erythrocyte sedimentation rate was 30 $\mathrm{mm}$ in the first hour, and C-reactive protein was $2.7 \mathrm{mg} / \mathrm{dl}$. Blood cell counts, serum and urine chemistry, immunoglobulins, complements, angiotensin converting enzymes, CSF, and EEG were normal. Antinuclear antibody, anti-DNA, SS-A, SS-B, anti-ribonucleoprotein, anti-Smith, lupus erythematosus test, anti-treponema pallidum antibodies, and immune complexes were all negative. AntiHTLV-I antibody was positive at 1:512 in serum by the PA method whereas it was not detected in CSF. A quantitative polymerase chain reaction analysis of HTLV-I pX DNA ${ }^{8}$ showed that four copies of HTLV-I genome were present per 100 peripheral blood mononuclear cells. Other viral antibody studies were unremarkable in either the serum or CSF. Culture of CSF was negative. A spontaneous proliferation of peripheral blood mononuclear cells increased significantly to 2994 whereas the stimulation index to PHA and ConA decreased to 24.7 and 21.5 respectively. The CD4 + cells in peripheral blood increased to $55 \cdot 5 \%$, whereas the CD8 + cells were within the normal range $(22.6 \%)$ based on flow cytometry.

A chest radiograph showed strand and acinar shadows in both lower lung fields, and body CT disclosed fibrobullous changes throughout the lungs. Brain MRI disclosed diffuse thickening and gadolinium enhance-

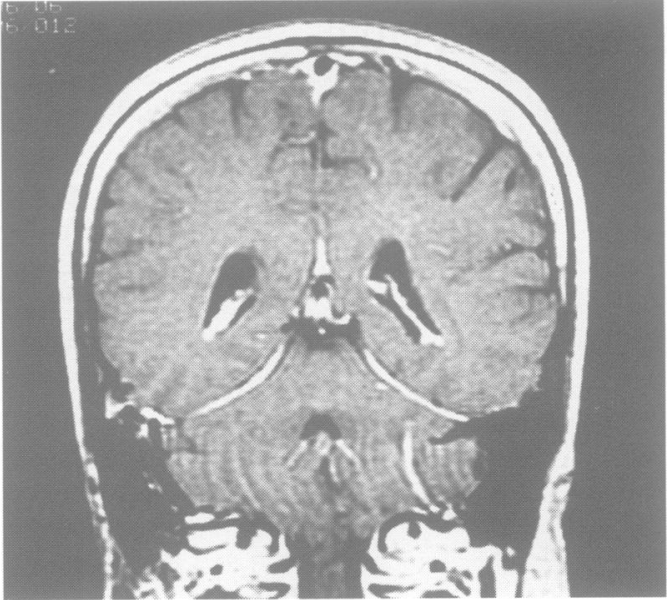

(B)

ment of dura mater in the falx, skull base, and cerebellar tentorium. No abnormal accumulation was seen on gallium scintigraphy of the head.

\section{Discussion}

Neither of these patients showed any underlying infectious or autoimmune disease as a cause for chronic hypertrophic cranial pachymeningitis except for the HTLV-I infection. The link between HTLV-I infection and HAM/TSP has been established. ${ }^{12}$ In this condition, immunological aberrance ${ }^{9}{ }^{10}$ as well as increased HTLV-I proviral DNA load ${ }^{8}$ are characteristic. Both of our patients showed an increased spontaneous proliferation and decreased mitogenic response of peripheral blood mononuclear cells in addition to the increase of helper/inducer $T$ cells in peripheral blood. All of these changes are commonly found in HAM/TSP. ${ }^{10}$ Moreover, the increase of HTLV-I proviral DNA load as well as the presence of antibody against HTLV-I in CSF strongly suggest an association of HTLVI infection with pachymeningitis in patient 1 . An autoimmune or inflammatory process is likely to be operative in this case, as corticosteroids produced beneficial effects.

Patient 2 showed an intermediate value of HTLV-I proviral DNA load between the patients with HAM/TSP and non-HAM/TSP carriers without inflammation. ${ }^{8}$ This may be explained by the long standing history of the disease, as it has been shown that HTLV-I proviral DNA load decreased as the disease duration lengthened.$^{8} \mathrm{His}$ condition was complicated by bronchopneumonia of unspecified aetiology. This may also be related to HTLV-I infection, as the lungs are often involved in HTLV-I infection. ${ }^{5}$ Although the relation between HTLV-I infection and pachymeningitis was less clear in patient $2, \mathrm{HTLV}-\mathrm{I}$ was at least considered as an aggravating factor for the disease through its inappropriate immune activation.

Interestingly, cytokines, such as tumour necrosis factor- $\beta$, secreted from the HTLV-Iinfected cell lines greatly enhance the proliferation of the fibroblasts. ${ }^{11}$ Therefore, HTLV-I 
can contribute to the development of hypertrophic pachymeningitis, firstly through aberrant immune activation, and secondly through fibroblast growth promoting effects. It is notable that the considerable fibrous thickening of the meninges were commonly found in HAM/TSP. ${ }^{12}$ We therefore suggest that chronic hypertrophic pachymeningitis can occur as a part of multiorgan involvement in HTLV-I infection, and that the HTLV-I carrier state should be examined in this condition in HTLV-I endemic areas. Alternatively, in HTLV-I carriers, especially in those with inflammatory diseases who present with either headache or cranial neuropathy, a gadolinium enhanced MRI is warranted to look for pachymeningitis.

1 Gessain A, Barin F, Vernant JC, et al. Antibodies to human T-lymphotropic virus type-I in patients with tropical spastic paraparesis. Lancet 1985;2:407-10.

2 Osame M, Matsumoto M, Usuku $\mathrm{K}$, et al. Chronic progressive myelopathy associated with elevated antibodies to human T-lymphotropic virus type-I and adult T-cell leukemia like cells. Ann Neurol 1987;21:2117-22.

3 Nishioka K, Maruyama I Sato K, al Cir tory arthropathy associated with HTLV-I. Lancet 1989; tory a

4 Morgan OS, Rodgers-Johnson P, Mora C, Char G. HTLV$I$ and polymyositis in Jamaica. Lancet 1989;2:1184-7.

5 Sugimoto $M$, Nakashima $H$, Watanabe $S$, et al. T-lymphotropic alveolitis in HTLV-I associated myelopathy. Lancet 1987;2:1220.

6 Mochizuki M, Watanabe T, Yamaguchi K, et al. HTLV-I uveitis: a distinct clinical entity caused by HTLV-I. $\mathfrak{f p}_{p} \mathfrak{f}$ Cancer Res 1992;83:236-9.

7 Luis Cartier R, Jose Luis Castillo C, Jose Gabriel Cea M, Rogue Villagra C. Chronic dacryosialadenitis in HTLV-I associated myelopathy. I Neurol Neurosurg Psychiatry 1995;58:224-6.

8 Kira J, Koyanagi Y, Yamada T, et al Increased HTLV-I proviral DNA in HTLV-I-associated myelopathy: a proviral DNA in HTLV-I-associated myelopathy: a quantitative polym

9 Itoyama Y, Minato S, Kira J, et al. Spontaneous proliferation of peripheral blood lymphocytes increased in patients with HTLV-I-associated myelopathy. Neurology 1988;38:1302-7.

10 Itoyama Y, Kira J, Fujii N, et al. Increases in helper induce $T$ cells and activated $T$ cells in HTLV-I-associated myelopathy. Ann Neurol 1989;26:257-62.

11 Yu F, Itoyama Y, Kira J, et al. TNF- $\beta$ produced by human $T$ lymphotropic virus type I-infected cells influences the proliferation of human endothelial cells and fibroblasts. $f$ Immunol 1994;152:5930-6.

12 Iwasaki $\mathrm{Y}$. Pathology of chronic myelopathy associated with HTLV-I infection (HAM/TSP). F Neurol Sci 1990; 96:103-23. 